\title{
Design of high precision pressure-resistance type pressure sensor detecting system
}

\author{
Xueliang Zhao ${ }^{1, \text { a }}$, Shuren $\mathrm{Gao}^{2}$, Tao Wang ${ }^{2}$ and Yingping Guo ${ }^{1}$ \\ ${ }^{1}$ Center For Hydrogeology And Environmental Geology Survey,CGS, Key Laboratory of Geological Environment \\ Monitoring Technology, Ministry of Land and Resources, Baoding of Hebei Province China \\ ${ }^{2}$ Petroleum Production Engineering Research Institute, Huabei Oilfield Company, CNPC, Renqiu China
}

\begin{abstract}
The high precision detecting circuit is designed for the KELLER 3L sensor which is the pressure-resistance pressure sensor, and the water level long time monitoring of groundwater is accomplished. Through designing the power management circuit, the pressure sensor driver circuit, the signal conditioning circuit and temperature compensation method, the problem of zero drift and temperature drift is solved. The experiment shows that the pressure detecting instrument has the very good application prospect with the advantages of small size, low power consumption, high precision and so on.
\end{abstract}

Keywords: pressure-resistance type pressure sensor; water level monitoring; detecting circuit; groundwater.

\section{Introduction}

The high precision monitoring of groundwater level is very important for the groundwater resources management and groundwater environment quality. The measurement of water level need use the pressure sensor. The pressure sensor includes float-type and pressure-resistance type generally. Along with the development of the science and technology, automation, online-monitoring, intelligence and high precision are the trend of groundwater level monitoring. The float-type pressure monitoring instrument has not matched the demand because of the disadvantages for example low precision, poor sensitivity and so on. The pressure-resistance pressure sensor can transform the water level into voltage signal, and is very easy to accomplish the long-time online high precision monitoring. However, the pressure sensor driver circuit and the temperature compensation are the key factors for the measurement precision. Aim at the groundwater level demand the power management circuit, the pressure sensor driver circuit and temperature measuring circuit are designed based on the pressure-resistance pressure sensor. The measurement accuracy is improved greatly, and the water level monitoring instrument is very suit to the demand at present.

\section{The KELLER 3L pressure sensor}

The KELLER 3L pressure sensor is a pressure-resistance pressure sensor which is manufactured by diaphragm technology based on laser welding. It's size is $\Phi 9.5 \times 4.2 \mathrm{~mm}$, and the pressure scale is 20 to

a Corresponding author : zxl-1229@163.com 
200 bar. The accuracy is $0.25 \% \mathrm{FS}$. The bridge circuit is included inside the sensor. It is very easy to be integrated with the detecting circuit and instrumentation housing. The groundwater level monitoring instrument is suit to be installed inside the monitoring wells to long-time monitor the groundwater situation based on the $3 \mathrm{~L}$ pressure sensor. The internal equivalent circuit diagram of $3 \mathrm{~L}$ pressure sensor is shown in the figure 1.

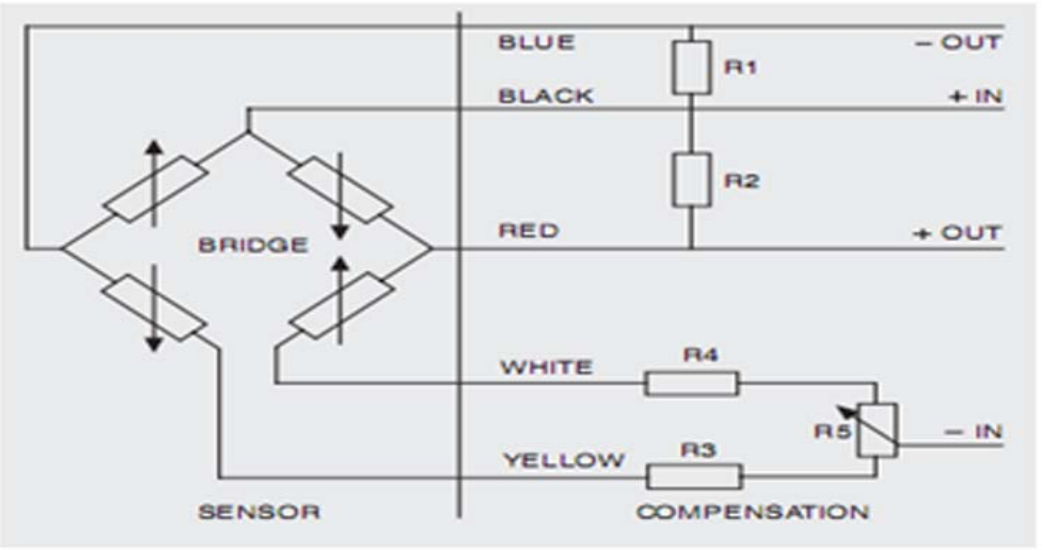

Figure 1. The internal equivalent circuit diagram of $3 \mathrm{~L}$ pressure sensor

\section{Hardware design}

The MSP430F548[5] is adopted as the main processor. The whole instrument includes six parts: the processor minimum system, the constant current source pressure sensor driver circuit, the constant current source temperature electrode driver circuit, the signal transmission circuit, the power manage circuit and the host computer. The processor minimum system controls other parts to work orderly and collect the pressure sensor analog signal and temperature sensor signal. The pressure-resistance pressure sensor is drove by the constant current source pressure sensor driver circuit. The temperature sensor is drove by the constant current source temperature electrode driver circuit. The different part's supplies are managed by the power manage circuit. The monitoring result data is transmitted to the host computer by the 485 bus. The water level data and water temperature data are saved and displayed by the host computer. The whole block diagram is shown in the figure 2 .

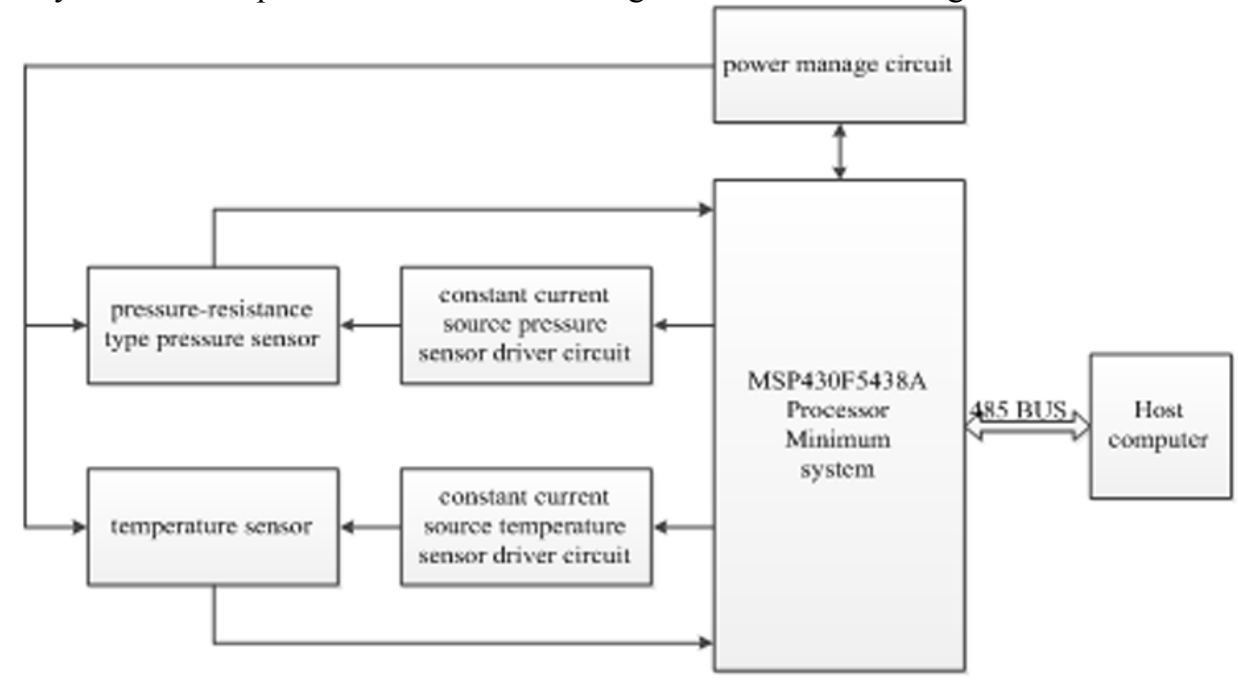

Figure 2. The whole block diagram 


\subsection{MSP430F5438 and ADC12 module}

The MSP430F5438 is the 16 bit ultra-lowpowersinglechip of TI based on the RISCarchitectures. The current can achieve $\mathrm{u}$ a level in the conditionofultra-low powerconsumption by the power management module of MSP430F5438. The multichannel and high precision ADC12 module is integrated into the MSP430F5438 [5]. It includes ADC core, $2.5 \mathrm{~V} / 1.5 \mathrm{~V}$ reference voltage generator and a variety of clock source etc. The ADC12 module can meet manydatacollectionapplications, so that the hardware design can been greatlysimplified. The ADC12 block diagram is shown in figure 3 .

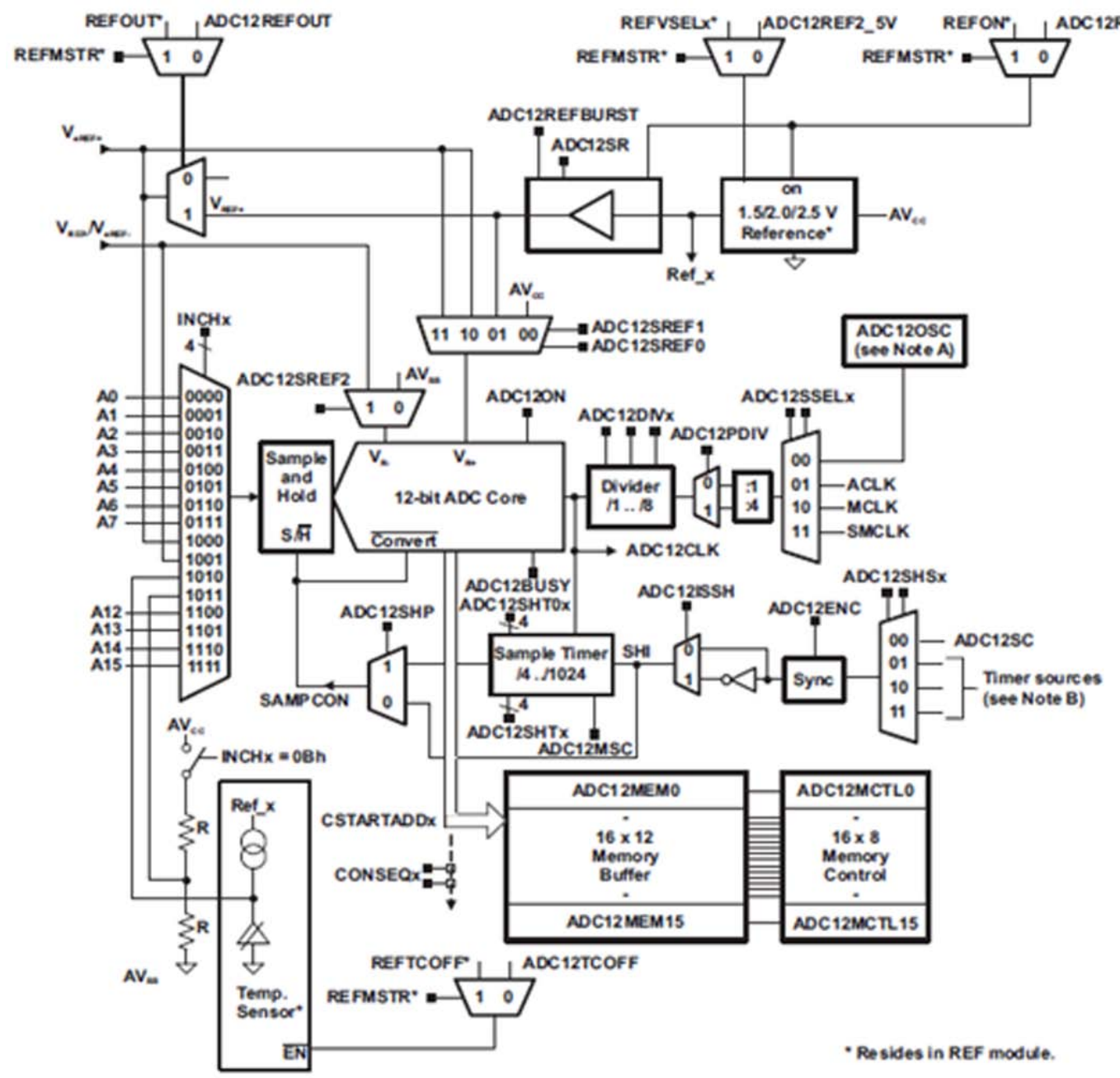

Figure 3. The ADC12 block diagram

The A0 channel and A1 channel are chosen to collect the signal of the thermostat sensor and the pressure-resistance type pressure sensor. The registers are set as follow.

ADC12CTL2=ADC12RES_2;

$\mathrm{ADC} 12 \mathrm{ICTL} 0=\mathrm{ADC} 12 \mathrm{ON}+\mathrm{ADC} 12 \mathrm{SHT} 0$

$\mathrm{ADC} 12 \mathrm{CTL} 1=\mathrm{ADC} 12 \mathrm{SSEL} 1+\mathrm{ADC} 12 \mathrm{DIV} 0+\mathrm{ADC} 12 \mathrm{SHP}+\mathrm{ADC} 12 \mathrm{CONSEQ} 0$

\subsection{The constant current source pressure sensor driver circuit}

The pressure-resistance pressure sensor can be drove by the constant current source and the constant voltage source. The constant current source which is compared with constant voltage source is benefit 
to reduce the temperature influence. The constant current source driver circuit is designed to let the pressure-resistance type pressure sensor work normally [1]. The circuit is shown in the figure 4 .

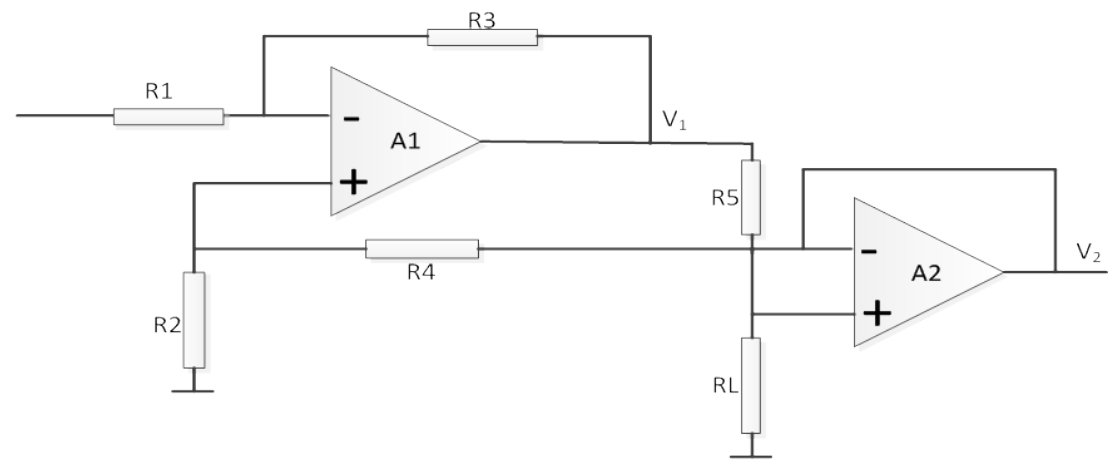

Figure 4. The constant current source driver circuit

The constant current source driver circuit is constructed by operational amplifiers A1, A2 and resistances. The operational amplifiers A1 and A2 use LM358. The current formula is shown as follow:

$$
\mathrm{I}=-\mathrm{V} 0 \mathrm{R} 3 / \mathrm{R} 1 \mathrm{R} 5 \quad(\mathrm{R} 1 \times \mathrm{R} 4=\mathrm{R} 2 \times \mathrm{R} 3)
$$

The current which is generated by Fig. 4 is $1 \mathrm{~mA}$ according to the KELLER 3L pressure sensor's demand.

\subsection{Pressure sensor signal conditioning circuit}

The output resistance of pressure-resistance type pressure sensor is very high. Therefore, the pressure sensor signal conditioning circuit need have very bigger input resistance than the output resistance to guarantee the normal work of pressure sensor [2]. The pressure sensor signal conditioning circuit is shown in the figure 5 .

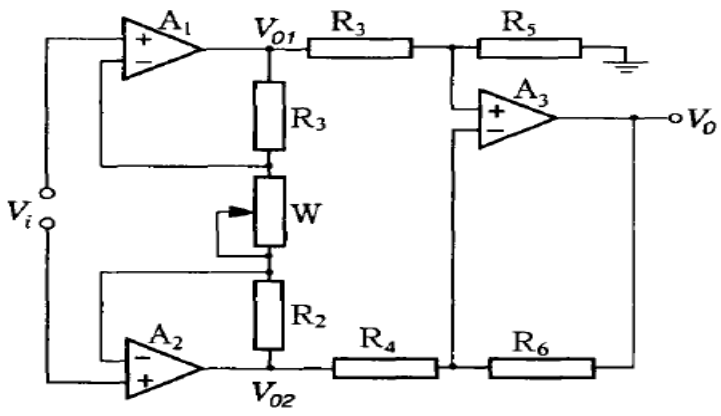

Figure 5.The signal conditioning circuit

The first stage co phase parallel connection differential amplifier circuit is constructed by the operational amplifiers A1 and A2. The second stage amplifier circuit is designed by A3 to improve the gain. The signal conditioning circuit has high input resistance, CMRR and open-loop gain with small offset current and noise, and can inhibit the common-signal interference effectively. The value of R1, $\mathrm{R} 2, \mathrm{R} 3$ and $\mathrm{R} 4$ are R. The value of R5 and R6 are Rf. The value of output voltage can be adjusted by the Adjustable resistance $\mathrm{W}$. The value of output voltage is calculated according to the formula as follow.

$$
V_{o}=-\left(R_{f} / R\right)[1+2 R / W] V_{i}
$$




\section{Software design}

The system use the methodofmodularization, and the software program is written by $\mathrm{C}$ programming language in the IAR Embedded Workbench. The whole program include five parts: the main program, the supply management program, the pressure sensor signal acquisition program, the temperature measurement program, data handle program [3], the 485 communication program. The system program flow chart is shown in figure 6.

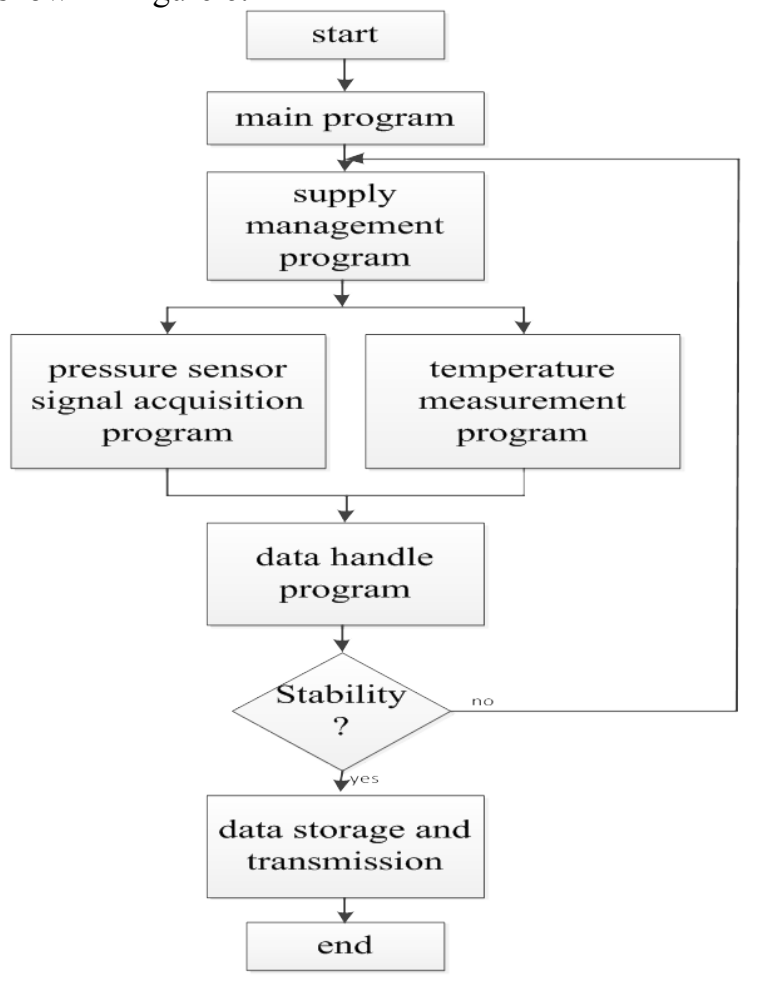

Figure 6. The system program diagram

\subsection{Temperature compensation method}

The temperature is the main reason which influences the pressure measurement accuracy. So the temperature compensation must be carried out. The least square method is used to build the two non-linear function relationship including the relation of pressure sensor output voltage-pressure and the relation of temperature-Curve coefficient [4].The specific temperature compensation method includes three steps. Firstly, the curve equations need been built in different temperatures through experiments. Secondly, the curve equations are bulit between Curve coefficient which is in the first step and temperature. Finally, the final compensation equation is built through the first step and the second step.

The DS18B20 which is a kind of digital temperature sensor is adopted to measure the groundwater temperature. The DS18B20 measurement circuit is very simply, and can minimum the whole hardware design the complexity.

\section{The application result}

The pressure-resistance type Pressure Sensor Detecting system's performance is certificated by the analogy device which can analogy groundwater environment for example water pressure, water 
temperature and water velocity of flow. The results are shown in table 1.

Table 1.Pressure sensor detecting system's performance experiment

\begin{tabular}{cccc}
\hline Temperature $\left({ }^{\circ} \mathrm{C}\right)$ & analogy pressor $(\mathrm{KPa})$ & measurement value $(\mathrm{KPa})$ & relative error $(\%)$ \\
0 & 100 & 99.80 & 0.20 \\
0 & 200 & 199.60 & 0.40 \\
& 300 & 299.34 & 0.66 \\
100 & 99.61 & 0.39 \\
\multirow{2}{*}{10} & 200 & 199.48 & 0.52 \\
& 300 & 299.30 & 0.70 \\
30 & 100 & 99.50 & 0.50 \\
& 200 & 199.35 & 0.65 \\
& 300 & 299.20 & 0.80 \\
& 100 & 99.36 & 0.64 \\
45 & 200 & 199.37 & 0.63 \\
& 300 & 299.20 & 0.80 \\
\hline
\end{tabular}

The table shows that the maximun relative error of pressor measurement system is $0.80 \%$ under the different temperature and pressure. The pressure measurement system is suit to be installed in the well to long-time online monitor the water level and water temperature with the advantages of small size, low power consumption, high precision and so on.

\section{Conclusion}

The high accuracy pressure-resistance type pressure sensor measurement system is achieved finally by using the MSP430F5438A processor, the constant current source driver circuit, the signal conditioning circuit and temperature compensation. The laboratoryexperiment is carried out, and the result has very good effect. The measurement system has board prospectsinapplication with the advantage of low-power dissipation, simple structure and highreliability.

\section{Acknowledgment}

This research is Supported by National Natural Science Foundation of China No.41303089; Geological Survey Project of China No.121201012000150010; The special public welfare industry research of The Ministry of land and resources in China N0.201411083-3.

\section{References}

1. Hu Yuanyuan, Wang Dajun. Intelligent Design of Pressure Sensor Temperature Compensation Based on ATmegal6[J]. Instnmient Technique and Sensor, 2010,10:9-11.

2. Cai Ying, Bi Peng. Pressure-resistance Type Pressure Sensor andDesign of Its Application Circuit[J]. Measuremence and Overhaul,2002,22(5):12-14.

3. Liu Peng, Yang Xueyou. Design of C Compensating Silicon Piezoresistive Sensor' Error based on MAX1452[J]. Instnmient Technique and Sensor, 2010,4:52-65.

4. Ni Xiuhui, Zhang Linlin, Ren Guoxing.Design of High Precision Thermistor TanperatureMeasuranent Based on MSP430[J]. Instnmient Technique and Sensor,2009, 3:101-120.

5. MSP430x5xx Family User's Guide [J/OL].2008,http://www.ti.com. 